\title{
Electromechanical Interplay in Deformable Dielectric Elastomer Networks
}

\author{
Noy Cohen and Gal deBotton* \\ Department of Mechanical Engineering, Ben-Gurion University, Beer Sheva 8410501, Israel
}

(Received 7 February 2016; published 20 May 2016)

\begin{abstract}
A systematic, statistical-mechanics-based analysis of the response of dielectric elastomers to coupled electromechanical loading is conducted, starting from the monomer level through the polymer chain and ending with closed-form expressions for the polarization and stress fields. It is found that the apparent response at the macrolevel is dictated by four microscopic parameters-the monomer type and polarizability and the chain length and density. Our analysis further reveals a new electrostrictive effect that either reinforces or opposes the polarization-induced deformation. The validity of the results is attested through comparisons with well-established experimental measurements of both the polarization field and the electrostrictive stress.
\end{abstract}

DOI: 10.1103/PhysRevLett.116.208303

Dielectric elastomers (DEs) are promising materials with a wide range of possible applications. These materials, that deform in response to electrostatic excitation, have been extensively investigated in the past 15 years [1,2]. Broadly speaking, the idea is to convert electrical energy into mechanical actuation by squeezing a DE medium between two oppositely charged electrodes (Fig. 1). In spite of attractive features such as a simple actuation mechanism, light weight, fast response time, and, above all, ability to undergo giant deformations, their development is hindered due to the low energy and the small forces they produce [2]. These shortcomings stem from the poor ratio between the dielectric and the mechanical properties in common soft elastomers.

Recent attempts to overcome these obstacles by enhancing the electromechanical coupling in DEs imply that it can be dramatically improved by tailoring their microstructures [2-8]. Motivated by these findings, the aim of this study is to progress along this path and introduce a novel model that enables us to accurately predict the electromechanical response of DEs in terms of the microstructural parameters at the polymer chain level. This model differs from existing ones, which are phenomenological in essence and are commonly based on the widely used ideal dielectric elastomer (IDE) model. According to the IDE model, the polarization linearly depends on the electric field, and the stress is the sum of a purely mechanical deformation-dependent term and an electrostatic stress that depends quadratically on the electric field $[1,8,9]$. While a few attempts to incorporate information concerning the network structure were made, these involved qualitative considerations that led to approximate or adjustable phenomenological models $[2,6,9]$. At present, systematic models that connect the microscale to the macroscale describe only either the electrical or the mechanical responses of DEs, but not the coupled one. Here we present a methodical, statistical-mechanics-based model that describes the coupled behavior of DEs and properly reduces to the appropriate uncoupled model when the DE is subjected to either electrical or mechanical loading.

Our analysis emanates from the first law of thermodynamics for DEs [10], where we assume that the energy stored in a polymer network can be expressed as the sum of the energies of the chains $[11,12]$. Locally, the electrical enthalpy of the monomers composing the chains is $u=-\mathbf{m}(\mathbf{E}) \cdot \mathbf{E}$, where $\mathbf{m}$ is the monomer electric dipole and $\mathbf{E}$ is the electric field. Taking the linear term of Taylor expansion series of $\mathbf{m}$ about $\mathbf{E}$ [11], we examine two types of monomers [13,14]. First, a uniaxial $(U)$ monomer whose dipole is aligned with the monomer direction

$$
\mathbf{m}=K_{U}(\mathbf{E} \cdot \hat{\boldsymbol{\xi}}) \hat{\boldsymbol{\xi}},
$$

[Fig. 2(a)]. Here, $\hat{\boldsymbol{\xi}}$ is the unit vector along the monomer and $K_{U}$ is the monomer polarizability (units $\mathrm{F} \cdot \mathrm{m}^{2}$ ). Second, a transversely isotropic (TI) monomer whose dipole is along the projection of the electric field on the plane perpendicular to the monomer direction

$$
\mathbf{m}=\frac{1}{2} K_{\mathrm{TI}}[\mathbf{E}-(\mathbf{E} \cdot \hat{\xi}) \hat{\xi}]
$$

with $K_{\mathrm{TI}}$ the polarizability of the TI monomer [Fig. 2(b)].

At the chain level, we adopt the affine deformation assumption according to which the deformations of the chain's end-to-end vectors ( $\mathbf{r}$ in Fig. 2) are governed by the

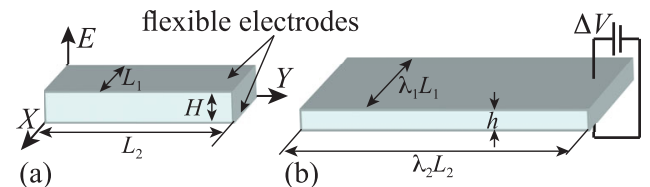

FIG. 1. A DE layer in its (a) unloaded and (b) electromechanically loaded configurations. 
(a)
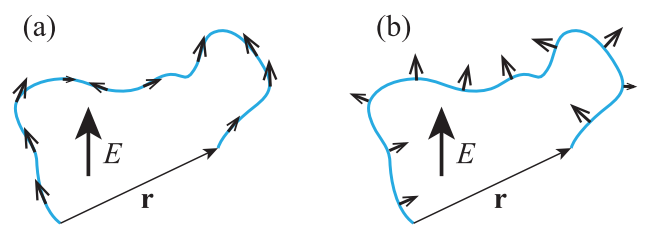

FIG. 2. Polymer chains with (a) uniaxial and (b) transversely isotropic monomers [13].

macroscopic deformation [15]. This enables us to impose three constraints on the conformations of the chains in terms of three Lagrange multipliers [16]. The first corresponds to the number of the monomers in the chain, the second corresponds to the prescribed electrical enthalpy of the chain, and the third Lagrange multiplier $\tau$, which is a vector, corresponds to the imposed deformation of the endto-end vector. Subjected to these constraints, we determine the entropy in terms of the most favorable chain conformation under the applied electromechanical loading [16]. In this chain,

$$
p(\hat{\boldsymbol{\xi}}, u)=\frac{1}{Z} \exp \left(\boldsymbol{\tau} \cdot \hat{\boldsymbol{\xi}}-\frac{u}{k \theta}\right)
$$

is the probability density function that a monomer is directed along $\hat{\xi}$ and its electrical enthalpy is $u$. Here, $k$ is the Boltzmann constant, $\theta$ is the temperature, and $Z=$ $\int \exp \left(\boldsymbol{\tau} \cdot \hat{\boldsymbol{\xi}}-\frac{u}{k \theta}\right) d \Gamma$ is the partition function, where $d \Gamma$ is a differential solid angle. The Lagrange multiplier $\boldsymbol{\tau}$ in Eq. (3) is extracted from the constraint

$$
n l \int \boldsymbol{\tau} p(\hat{\boldsymbol{\xi}}, u) d \Gamma=\mathbf{r}
$$

where $n$ is the number of the monomers in the chain and $l$ is the monomer length. Finally, assuming that the chains have an identical number of monomers and a random referential distribution [15], we integrate over all the chains in the DE and derive the macroscopic polarization and stress fields in the deformed dielectric [17].

Hereafter, we examine the common setting of a thin DE layer between two electrically attracted flexible electrodes. We restrict the analysis to a deformation range substantially smaller than the chain's extensibility limit $[5,6,8,9,18-20]$. This, in turn, allows us to extract an explicit expression for $\tau$ from Eq. (4). In its unloaded state, the layer dimensions are $L_{1}$ and $L_{2}$, and its thickness is $H$ [Fig. 1(a)]. When loaded, the layer dimensions are held fixed at $\lambda_{1} L_{1}$ and $\lambda_{2} L_{2}$, and the thickness of the incompressible layer is $h=\frac{H}{\lambda_{1} \lambda_{2}}$ [Fig. 1(b)]. The electric potential $\Delta V$ between the electrodes induces the electric field $E=-\lambda_{1} \lambda_{2}(\Delta V / H)$ along the $E$ axis.
The predicted polarization along the electric field is

$$
\begin{aligned}
& P_{E}\left(\lambda_{1}, \lambda_{2}, E\right) \\
& \quad=N_{0} K E \eta\left(\frac{n}{2}+c^{2} \frac{\left[2(a-1)^{2}-\lambda_{1}^{2} \lambda_{2}^{2}\left(\lambda_{1}^{2}+\lambda_{2}^{2}\right)\right](b-a)}{24 \omega^{2} \lambda_{1}^{2} \lambda_{2}^{2}}\right),
\end{aligned}
$$

and the stress in the transverse direction is

$$
\sigma_{X}\left(\lambda_{1}, \lambda_{2}, E\right)=\sigma_{X}^{m}-E P_{E}-\varepsilon_{0} E^{2},
$$

where

$$
\sigma_{X}^{m}=N_{0} k \theta \frac{c}{3}\left(\lambda_{1}^{2}-\frac{1-a}{\lambda_{1}^{2} \lambda_{2}^{2}}\right)
$$

is referred to as the mechanical component of the stress, $\omega=\sqrt{K / k \theta} E, N_{0}$ is the number of chains per unit referential volume, and $\varepsilon_{0}=8.85 \times 10^{-12} \mathrm{~F} \cdot \mathrm{m}^{-1}$ is the permittivity of the vacuum. The expression for $\sigma_{Y}$, the stress in the $Y$ direction, is identical with a switch of the roles of $\lambda_{1}$ and $\lambda_{2}$. In the above expressions, $K=K_{U}$ and $K=K_{\mathrm{TI}}$ for dielectrics with uniaxial and transversely isotropic dipoles, and $\eta, a, b$, and $c$ are even functions of $E$ only. For a dielectric with uniaxial dipoles, $\eta=(\omega-D) /\left(\omega^{2} D\right)$, $a=(3 \eta-2) /(2 \eta), b=\omega^{2}[1-(\eta / 2)], c=4 /(2-\eta)$, and $D=\int_{0}^{\omega} e^{t^{2}-\omega^{2}} d t$ is the Dawson integral. For a dielectric with transversely isotropic dipoles,

$$
\eta=1-\frac{1}{\omega^{2}}+\sqrt{\frac{2}{\pi}} \frac{\exp \left(-\frac{\omega^{2}}{2}\right)}{\omega \operatorname{Erf}\left(\frac{\omega}{\sqrt{2}}\right)},
$$

$a=(2 / \eta)-3, b=\omega^{2}(\eta-1)$, and $c=(2 / \eta)$. Note that $\eta$ is a smooth monotonic function of $\omega$ varying from $\eta(0)=\frac{2}{3}$ to $\eta=2$ as $\omega \rightarrow \infty$ for dielectrics with uniaxial dipoles and in the range from $\frac{2}{3}$ to 1 for those with TI dipoles.

Before proceeding, four observations are in order. (i) In the limit of small electric fields and moderate deformations, Eq. (5) recovers the IDE model $P_{E}=\chi_{0} \varepsilon_{0} E$, where $\chi_{0}=$ $\left(n N_{0} / 3\right)\left(K / \varepsilon_{0}\right)$ is the elastomer initial susceptibility. (ii) The incompressibility assumption requires the addition of a pressurelike workless term to the stress whose value is extracted from the boundary condition. Thus, $\sigma_{X}$ in Eq. (6) incorporates a pressure term corresponding to a zero traction boundary condition on the electrodes (i.e., $\sigma_{E}=0$ ). Accounting for this term, in the limit of purely mechanical loading $\sigma_{X}^{m}$ recovers the well-known neo-Hookean model with initial modulus $\mu=N_{0} k \theta[6,15]$. Moreover, by setting $\eta, a$, and $c$ to their initial values and taking only the first term for $P_{E}$, under coupled electromechanical loading Eq. (6) recovers the IDE model. (iii) The contour length of a chain is $n l$, whereas the length of its end-to-end vector in the unloaded state is $\sqrt{n} l$ [15]. Thereby, the lockup stretch of 
the chain is $\lambda_{c}=\sqrt{n}$ [6]. (iv) When the layer thins under mechanical loading, then, according to the affine deformation assumption, in the deformed state the sum of the dipole projections in the direction of the electric field will decrease in chains with uniaxial monomers. Therefore, in these networks the susceptibility and the electromechanical coupling will diminish. For chains with TI monomers, the situation is reversed, and both the susceptibility and the coupling improve.

These observations enable us to deduce $n, N_{0}, K$, and the monomer type from measurements of $\lambda_{c}, \mu, \chi_{0}$, and the trend of the polymer response relative to the IDE model. Thereby, we infer that the VHB-4910 monomers are uniaxial and for this material $\lambda_{c}=9.6, \mu=73 \times 10^{3} \mathrm{~Pa}$, and $\chi_{0}=3.7$ [21]. The Elastosil monomers are of the TI type with $\lambda_{c}=7, \mu=342 \times 10^{3} \mathrm{~Pa}$, and $\chi_{0}=1.7$ [22].

Shown in Fig. 3 are four sets of experimentally measured results for VHB-4910 together with the predicted susceptibility $\chi=\left(P_{E} / \varepsilon_{0} E\right)$ according to Eq. (5). Following the experimental setups, $\chi$ is determined with $\lambda_{1}=\lambda_{2}=\lambda$ and shown versus the planar stretch $\lambda^{2}$. Kofod et al. [18] conducted the measurements at a frequency of $0.1 \mathrm{~Hz}$ and used metal conductor electrodes with carbon black. McKay, Calius, and Anderson [19] used gold and Nyogel electrodes. Wissler and Mazza [20] used gold plungers, and measurements were done at $100 \mathrm{~Hz}$. Equation (5) accurately reproduces the measurements with metallic electrodes $[18,19]$. In the other two cases, the low values of the measured susceptibilities can be attributed to the highly resistive Nyogel electrodes [19] and the high frequency at which the experiments were conducted [20].

Next, we examine the model predictions for the stress. Figures 4(a) and 4(b) show the measurements carried out by Pelrine, Kornbluh, and Kofod [23] and Wissler and Mazza [20] with VHB-4910 samples, respectively. Figure 4(c) shows results for Elastosil samples by Kofod and Sommer-Larsen [22]. Following the experimental setups, we fix the deformation and determine the difference between the stresses without and with electrical excitation. Thus, shown in Fig. 4 is the difference

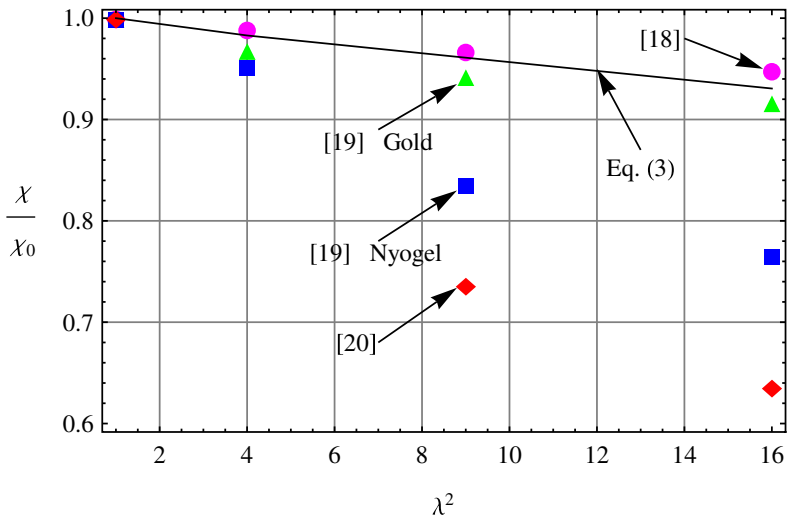

FIG. 3. Normalized susceptibility versus deformation.
$\sigma_{X}\left(\lambda_{1}, \lambda_{2}, 0\right)-\sigma_{X}\left(\lambda_{1}, \lambda_{2}, E\right)$ versus the electric field for the reported stretches and materials. In all three cases, there is a fine agreement between the outcomes of the analysis and the experiments.

Also shown in Fig. 4 are the curves for the quadratic electrostatic stress $\left(\chi_{0}+1\right) \varepsilon_{0} E^{2}$ according to the IDE model. This model overestimates the results measured for the VHB-4910 and vice versa for the Elastosil. Thereby, according to observation (iv) above, we assume uniaxial and TI monomers for the VHB-4910 and the Elastosil, respectively. The error of the IDE model relative to both the present analysis and the experimental measurements can be as large as $20 \%$.

An important outcome of our analysis concerns the dependence of the mechanical component of the stress $\sigma_{X}^{m}$ on the electric field. Thus, while the influence of the deformation on the polarization was phenomenologically
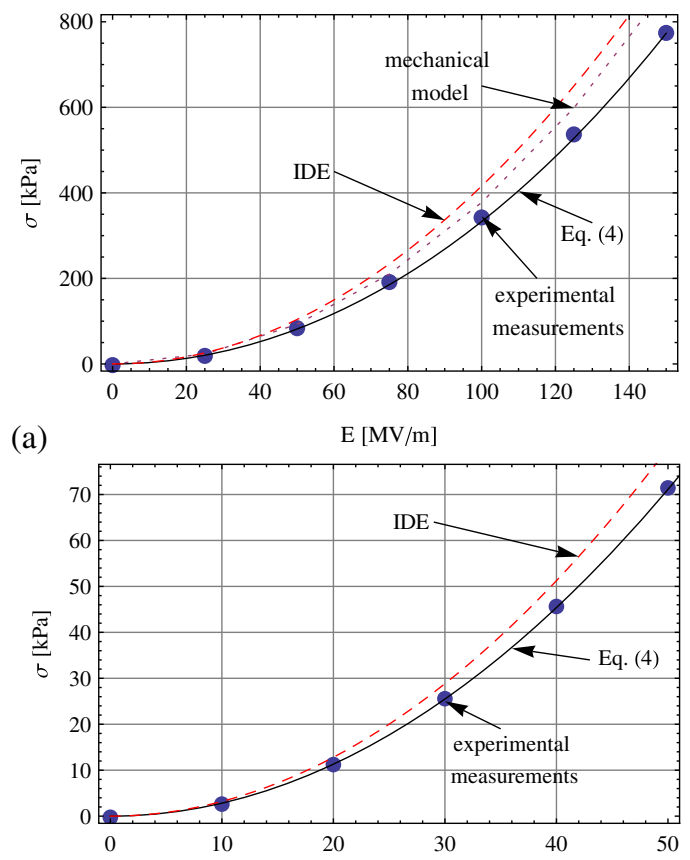

(b)

$\mathrm{E}[\mathrm{MV} / \mathrm{m}]$

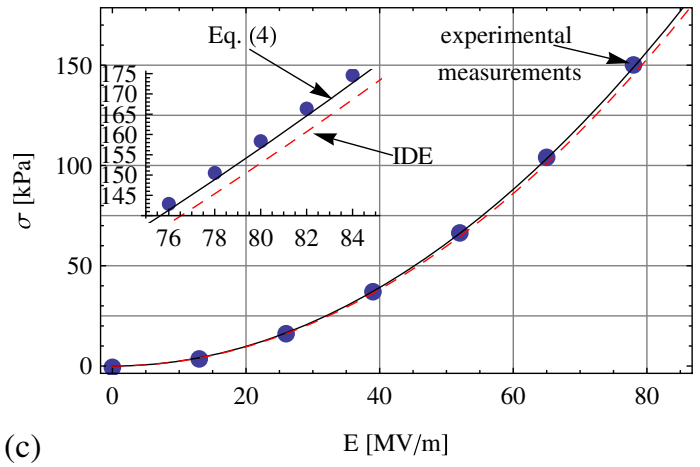

FIG. 4. Stress versus electric field. Analytical predictions and experimental measurements of (a) Pelrine et al. [23], (b) Wissler and Mazza [20] and (c) Kofod and Sommer-Larsen [22]. 
(a)

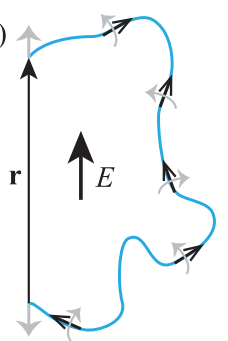

(b)

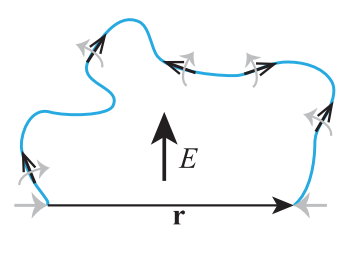

FIG. 5. Schematic description of the tendency of uniaxial dipoles to align along the electric field and the resulting forces on the end points of two chains whose end-to-end vectors are (a) along and (b) perpendicular to the electric field.

tackled in previous works [9], the countereffect was neglected. This electrostrictive effect can be explained in terms of the kinematic constraint imposed on the chain's end-to-end vectors [17]. In a way of an example, consider a chain with uniaxial monomers whose end-to-end vector is along the electric field [Fig. 5(a)]. The uniaxial monomers tend to line up in order to align their dipole with the electric field, and their combined motion prompts elongation of the end-to-end vector. At the macrolevel, this leads to a thickening of the layer, hence opposing the deformation induced directly by the electric attraction between the electrodes. In a chain whose end-to-end vector is perpendicular to the electric field, the tendency of the monomers to align with the electric field prompts a shortening of the chain and hence, once again, a thickening of the layer due to Poisson's effect [Fig. 5(b)]. This reasoning can be easily extended to any chain with uniaxial dipoles. In DEs with TI dipoles, the situation is reversed, and this electrostrictive effect acts to further squeeze the layer. Thus, this effect may reinforce or attenuate the electrostatic stress component $E P_{E}$ in Eq. (6). To quantify this effect, in Fig. 4(a), we added the short-dashed curve denoted mechanical model, resulting from the replacement of $\sigma_{X}^{m}$ in Eq. (6) with the purely mechanical term $\mu\left[\lambda_{1}^{2}-\left(\lambda_{1} \lambda_{2}\right)^{-2}\right]$ of the IDE model. It is evident that the contribution of this new electrostrictive term is substantial, and in this case its negligence results in a $13 \%$ error.

We conclude this Letter by recalling that the weak coupling between the electrostatic and the mechanical fields in currently available DEs motivated this study. To overcome this limitation, which is the main obstacle to widespread applications of these materials, the interplay between the origins of the electromechanical coupling at the monomer level and its apparent expression at the macrolevel, through the DEs chain network, must be analyzed. Accordingly, the goal of this Letter is to introduce a novel, statistical-mechanics-based model that accurately predicts the coupled response of DEs by elevating relevant information from the monomer and chain levels to the macroscopic level. Indeed, we obtain closed-form expressions for the stress and the polarization in terms of two parameters that are associated with the monomers (type and polarizability) and two parameters of the chains (length and density). The model demonstrates that the high electric fields that are being used to obtain meaningful deformations have a substantial influence on both the microstructure and the response of the polymer. Moreover, we reveal a new electrostrictive effect that is associated with the tendency of the monomers to align their dipole with the electric field and the kinematic constraint imposed on the chain's end-to-end vectors. We also certify our predictions against well-established sets of experimental results. Finally, note that the procedure followed in this work can be applied to characterize the behaviors of DEs with more complicated structures, such as ones with highly polarized side molecules that are attached to the primary chain network [2]. Thus, it provides a methodology to optimize the performance of DEs through enlightened enhancement of their microstructure. In this regard our analysis indicates that from a practical viewpoint DEs with TI dipoles are preferable, since the electrostrictive effect due to the kinematic constraint enhances the polarizationinduced stress, whereas in DEs with uniaxial dipoles this effect diminishes the overall deformation.

This work was supported by the Israel Science Foundation founded by the Israel Academy of Sciences and Humanities (Grant No. 1246/11).

*debotton@bgu.ac.il

[1] R. Pelrine, R. Kornbluh, Q.-B. Pei, and J. Joseph, Science 287, 836 (2000).

[2] F. B. Madsen, A. E. Daugaard, S. Hvilsted, and A. L. Skov, Macromol. Rapid Commun. 37, 378 (2016).

[3] C. Huang, Q. M. Zhang, G. deBotton, and K. Bhattacharya, Appl. Phys. Lett. 84, 4391 (2004).

[4] S. Rudykh, A. Lewinstein, G. Uner, and G. deBotton, Appl. Phys. Lett. 102, 151905 (2013).

[5] Z. Suo and J. Zhu, Appl. Phys. Lett. 95, 232909 (2009).

[6] X. Zhao and Z. Suo, Phys. Rev. Lett. 104, 178302 (2010).

[7] B. Kussmaul, S. Risse, G. Kofod, R. Waché, M. Wegener, D. N. McCarthy, H. Krüger, and R. Gerhard, Adv. Funct. Mater. 21, 4589 (2011).

[8] A. Tutcuoglu and C. Majidi, Appl. Phys. Lett. 105, 241905 (2014).

[9] X. Zhao and Z. Suo, J. Appl. Phys. 104, 123530 (2008).

[10] R. M. McMeeking, C. M. Landis, and S. M. A. Jimenez, Int. J. Nonlinear Mech. 42, 831 (2007).

[11] T. Blythe and D. Bloor, Electrical Properties of Polymers, 2nd ed. (Cambridge University Press, Cambridge, England, 2008).

[12] N. Cohen, A. Menzel, and G. deBotton, Proc. R. Soc. A 472, 20150462 (2016).

[13] W. H. Stockmayer, Pure Appl. Chem. 15, 539 (1967).

[14] N. Cohen and G. deBotton, Eur. J. Mech. A 48, 48 (2014).

[15] L. R. G. Treloar, The Physics of Rubber Elasticity (Clarendon, Oxford, 1975). 
[16] T. L. Hill, An Introduction to Statistical Thermodynamics, Addison-Wesley Series in Chemistry (Addison-Wesley, Reading, MA, 1960).

[17] N. Cohen, K. Dayal, and G. deBotton, J. Mech. Phys. Solids 92, 105 (2016).

[18] G. Kofod, P. Sommer-Larsen, R. Kornbluh, and R. Pelrine, J. Intell. Mater. Syst. Struct. 14, 787 (2003).

[19] T. G. McKay, E. P. Calius, and I. A. Anderson, in Electroactive Polymer Actuators and Devices (EAPAD), edited by Y. Bar Cohen and T. Wallmersperger (International Society for Optics and Photonics, Bellingham, WA, 2009), Vol. 7287.

[20] M. Wissler and E. Mazza, Sens. Actuators, A 138, 384 (2007).

[21] M. Bozlar, C. Punckt, S. Korkut, J. Zhu, C. C. Foo, Z. Suo, and I. A. Aksay, Appl. Phys. Lett. 101, 091907 (2012).

[22] G. Kofod and P. Sommer-Larsen, Sens. Actuators, A 122, 273 (2005).

[23] R. Pelrine, R. Kornbluh, and G. Kofod, Adv. Mater. 12, 1223 (2000). 